
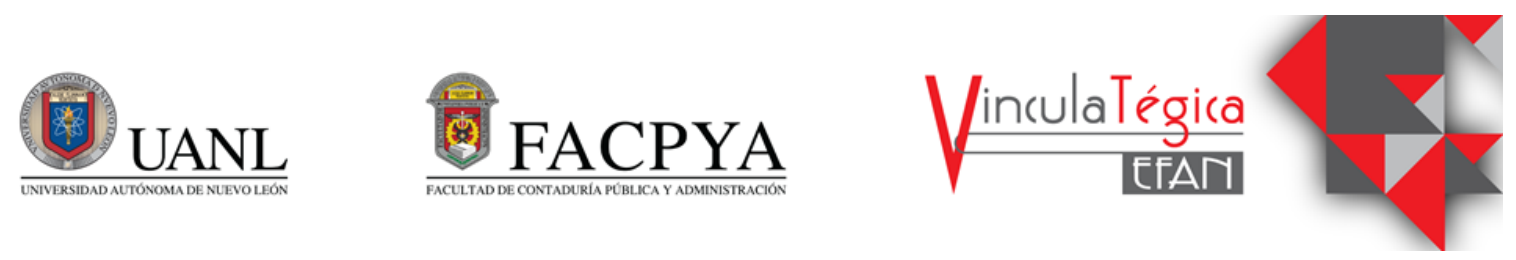

\title{
Los pequeños negocios y su consolidación en el mercado local
}

\author{
Héctor Enrique Escobar Olguín ${ }^{1}$, Dana Puente Sánchez ${ }^{2}$ y Itzel García Lara ${ }^{3}$ \\ ${ }^{1}$ Instituto Tecnológico Superior de San Pedro de las Colonias, hector.escobar@tecsanpedro.edu.mx, \\ El Tecnológico \#53, Col. El Tecnológico, 8721143195. \\ ${ }^{2}$ Instituto Tecnológico Superior de San Pedro de las Colonias, dana.puente18@logistica.tecsanpedro.edu.mx, \\ El Tecnológico \#53, Col. El Tecnológico, 8722957116. \\ ${ }^{3}$ Instituto Tecnológico Superior de San Pedro de las Colonias, itzel.garcia18@logistica.tecsanpedro.edu.mx, \\ El Tecnológico \#53, Col. El Tecnológico, 8721364985.
}

Información del artículo revisado por pares

Fecha de aceptación: junio-2021

Fecha de publicación en línea: diciembre-2021

DOI: https://doi.org/10.29105/vtga7.2-18

\section{Resumen}

La presente investigación se desarrolló en los municipios de San Pedro de las Colonias, Francisco I. Madero y algunas comunidades ejidales intermedias. Se encuestaron 122 negocios en total. La finalidad del estudio fue evidenciar los problemas u obstáculos (nivel de progreso, evaluación de su desempeño y la manera en que cada uno administra su negocio) que presentan los micro y pequeños negocios para consolidarse en el mercado local. Se empleó una metodología de carácter inductivo y explicativo-descriptivo. En un segundo momento se diseñó y validó un instrumento de 14 ítems, la escala de Likert fungió como una medida de selección de respuestas para facilitar su selección a los encuestados. En los resultados se observa que la mayoría de los negocios tienen una planeación parcialmente adecuada.

Palabras clave: Enseñanza universitaria, Comportamiento de la empresa, Estructura de mercado, estrategia empresarial $y$ funcionamiento del mercado, Generalidades

Códigos JEL: A22, D21, L1, L20
Abstract
The present investigation was developed in the municipalities of San Pedro de las Colonias and Francisco I. Madero, as well as in some intermediate ejido communities. A total of 122 businesses were surveyed. The purpose of the study was to show the problems or obstacles (level of progress, evaluation of their performance and the way each one manages their business) that micro and small businesses present to consolidate in the local market. An inductive, explanatory-descriptive methodology was used. In a second moment, a 14-item instrument was designed and validated; The Likert scale served as a measure of selection of responses to facilitate their selection to the respondents. The results show that most businesses have adequate planning. This has allowed them to have partially publicity. However, a good relationship with suppliers is not contemplated. The vast majority of businesses are dissatisfied in this regard.

Key words: University education, Company behavior, Market structure, business strategy and market operation, General

JEL Codes: A22, D21, L1, L20 


\section{INTRODUCCIÓN}

La presente investigación dará respuesta a los distintos inconvenientes a los que se pueden enfrentar los pequeños negocios. Después de una breve reflexión las problemáticas más relevantes resultan ser la planeación o gestión del negocio, la publicidad, y los arreglos con proveedores, teniendo entonces el siguiente objetivo: analizar cómo la falta de planeación en los negocios puede influir en la publicidad y en la relación con los proveedores. Es importante reconocer que las pequeñas empresas cuentan con una presencia significativo dentro de la economía nacional, a pesar de esto, es común que estos negocios tengan problemas al momento de intentar expandirse y entrar en un mercado mayor al que tienen disponible. Sin embargo, al ser pequeños negocios de giros variados, no todos presentan las mismas problemáticas. El enfoque principal de este artículo es mostrar un panorama y ofrecer alguna posible solución en relación con los problemas que enfrentan las empresas en la actualidad. Es aquí cuando surge la siguiente pregunta de investigación: ¿qué problemas o inconvenientes presentan los pequeños negocios para consolidarse en el mercado local? De manera empírica, se han identificado distintas problemáticas que afectan a los dueños de pequeños negocios, específicamente 14 inconvenientes: la falta de recursos económicos, la ubicación del negocio, la competencia, las ventas bajas, el espacio disponible, la economía de la ciudad, algunos permisos con el municipio, la falta de personal, no contar con un local propio, el mobiliario con el cuentan y la reactivación económica que sigue siendo frenada por la pandemia. Las tres principales problemáticas a los que se les puede dar solución son la planeación, la publicidad y los proveedores. La planeación resulta ser un inconveniente para los dueños de pequeños negocios debido a que la mayoría de ellos no cuenta con el conocimiento administrativo sobre cómo manejar un negocio. Sirve de gran ayuda ya que con una correcta planeación es más factible llevar a cabo una consolidación en el mercado. El elegir una estrategia de planeación implica que esta sea creativa, innovadora y lo más importante factible; es importante mencionar que las estrategias pueden verse afectadas por cuestiones externas que están fuera del negocio. La publicidad es un medio de paga para presentar y promocionar al público bienes y servicios de un patrocinador. La publicidad tiene un gran impacto en los clientes ya que a través de estos se les da a conocer y les incita a que lo adquieran. En los negocios encuestados, una de las principales problemáticas con la que coincidieron los dueños fue la falta de publicidad, ya que las empresas no le toman importancia a la publicidad, y esto ocasiona ventas bajas, problemas para darse a conocer e incluso, dificultades para promocionar sus productos. Hoy en día es de gran importancia para las pequeñas empresas, debido a que es un gran medio para para generar ventas; orienta a los pequeños negocios a dar a conocer productos y servicios a la sociedad.

Seleccionar el mejor proveedor en una tarea muy importante para las empresas. Es una decisión que dirige el rumbo de la misma. Estos deben de contar con requisitos que garanticen la realización de todos los procesos con la calidad solicitada por el cliente. La importancia y el compromiso depende de la confianza en las compras seleccionadas respetando los acuerdos y los costos expuestos por las dos partes para un desempeño que beneficie a ambos manteniendo inventarios de calidad, asegurando las necesidades con las que se cuenta satisfaciendo al cliente y dar mejores resultados. Sin proveedores no habría mercancía para vender, si se habla de una tienda se puede poner como ejemplo los proveedores de refrescos, frutas, artículos de limpieza etc. Con ellos la tienda tiene más variedad en sus productos por ende esto es más llamativo para los clientes y eso le conviene mucho a un negocio. La metodología consiste en una investigación de tipo cuantitativa, hecha mediante una encuesta estructurada con 14 ítems, realizada a 122 dueños de pequeñas empresas de distintos giros (en el que se destaca la venta de comida y misceláneas), en el municipio de San Pedro de las Colonias y ejidos a su alrededor, la encuesta fue aplicada en línea mediante un enlace, dando posteriormente una serie de resultados. La hipótesis de la investigación infiere que la falta de una planeación eficaz influye de manera negativa para la obtención de los objetivos de los negocios. Se busca comprobar la relación que existe entre una buena planeación de los negocios y los resultados al momento de esta ser aplicada en la publicidad y en el abastecimiento de mercancía con los proveedores.

\section{MARCO TEÓRICO}

\subsection{La planeación como eje rector.}

La planeación representa un papel importante en las decisiones de las pequeñas empresas. Sin ella, difícilmente se lograrán los objetivos identificados. Por lo tanto, (Reyes, 2019) argumenta que la planeación es uno de los elementos básicos para la continuidad de una empresa, como son los pequeños negocios. Por su parte, (Moreta, 2017) considera que es 
aplicable para todo tipo de empresas, sin embargo, para la pyme se ha tornado compleja por los constantes cambios que debe afrontar y sobre todo por la administración de tipo familiar que estas tienen. Dentro de los resultados obtenidos se hace mención de que los dueños de los pequeños negocios enfocan mayor atención a otro tipo de elementos y donde (Reyes, 2019), enfatiza que con frecuencia no se da el tiempo suficiente para atender de manera apropiada el proceso de planeación y gestión. Algunos de los encuestados hacían referencia a que contaban con conocimiento y experiencia en el giro de la empresa, la toma de decisiones es relativamente rápida, la cultura empresarial, entre otros, según (Reyes, 2019). Por su parte (Madrigal et al, 2015) opina de manera distinta al señalar que los aspectos desfavorables para este tipo de negocios son el sobrevivir el traspaso generacional de la organización, el temor a enfrentar riesgos limita el margen de acción y con ello la perdida de oportunidades.

Según (Correa, et al., 2010) la planeación se constituye en la base para la continuidad de las nuevas empresas que se crean a partir de planes de negocio. (Velasco, 2020), hace mención a que el problema que se plantea es distinguir los elementos actuales, ya sean internos o externos, que influyen o no en la administración de pequeños negocios y emprendimiento al considerar principalmente las cuatro prioridades para impulsar la competitividad. (Araya, 2017) argumentaba que en las empresas familiares, el cumplimiento de las metas por esos aspectos de sistemas es diferente, pues tienen mayor relevancia las preocupaciones emocionales, necesidades familiares y gestión de los cambios; para (Moreno \& Ortega, 2018) la planeación estratégica formal puede no ser adecuada para las pequeñas empresas ya que creen que un alto nivel de formalidad puede derivarse poca innovación y falta de creatividad y espontaneidad en los procesos. Para atende las áreas de oportunidad que mejorar, (Moreno \& Ortega, 2018), mencionan que los planificadores deben decidir, a partir de la situación dada, en qué medida es necesario y posible planificar desde abajo para asegurar que el punto de vista de los grupos destinatarios, sobre su proceso de desarrollo, sea adecuadamente incorporado a la planificación (Terrones, 2013). La Matriz de planeación del proyecto (mpp) sirve para informar a las instancias que asumen la responsabilidad del proyecto. La presentación de información en esta matriz puede realizarse también fuera del proceso participativo de planificación, si al hacerlo no se modifica unilateralmente lo acordado. Una mpp está expuesta al cambio, cuanto más profundo el nivel de planificación, tanto más frecuentemente se requieren modificaciones (Terrones 2013). El objetivo superior hace énfasis en lograr el aspecto global de la problemática planteada; mientras que el objetivo de desarrollo concentra la atención de todos los actores involucrados en el proyecto, en el proceso de desarrollo de los grupos destinatarios. Los proyectos se llevan a cabo para fomentar procesos de cambio que repercuten siempre en determinadas personas y organizaciones (Terrones 2013). El entusiasmo del empresario está enfocado principalmente en el manejo de los productos y servicios del negocio, de tal manera que con frecuencia no se da el tiempo suficiente para atender de manera apropiada el proceso de planeación y gestión (Reyes, 2019). La planeación estratégica es un proceso que requiere intencionalidad, para ello, es necesario que los beneficiarios del mismo, en este caso los empresarios, identifiquen los beneficios que pudieran obtener de llevar a cabo planeación estratégica en sus negocios (Moreno \& Ortega, 2018). Se establece que los gerentes desconocen sobre temas de planeación y prospectiva. El diseño de la estrategia se basa en la experiencia, por lo que no se documentan los diagnósticos, ni tampoco las acciones estratégicas adoptadas (Moreta, 2017). Respecto a los elementos para iniciar el negocio, se nota que las estrategias están en función del fundador de la empresa, que se caracteriza por el motivo que impulso a administrar un pequeño negocio, con base a una necesidad, deseo o demanda de los productos, esto depende en la etapa que se encuentre (Velasco, 2020). Las empresas hacen uso de la planeación estratégica con el fin de establecer los objetivos que se desean 
alcanzar, analizando su estado actual y las condiciones del entorno que le ayuden a determinar el rumbo que deberán tomar para el logro de sus metas.

(Velasco, 2020) argumenta que el crecimiento de los pequeños negocios se encuentra en una administración de la innovación, el conocimiento y el aprendizaje, donde conviene analizar la gestión de la competitividad empresarial de ellos.

\subsection{La publicidad, elemento valioso}

Según (Valdivia, 2015) la primera etapa del proceso de planificación estratégica de marketing la constituye el análisis de situación, realizando un estudio riguroso tanto de la situación externa de la empresa como de la situación interna. Para (Fernández, 2005), la publicidad potencia el consumo superfluo y sus mensajes "nos rodean, nos asfixian, nos confunden, nos divierten y, por supuesto, nos manipulan". (Cabezuelo, et al, 2012) argumenta que los duros tiempos que vive el sector publicitario español, se ha convertido en un eje fundamental para la toma de decisiones a la hora de poner en marcha una campaña de comunicación o de publicidad. La publicidad, aunque se ocupa principalmente de la promoción, se basa en decisiones administrativas sólidas, conjuntamente con las otras tres áreas de la mezcla de mercadotecnia, para que tenga éxito (Barillas, 2010). Según (Tellis \& Redondo, 2002) los anunciantes de publicidad deben emplear argumentos sólidos, porque los argumentos débiles podrían ser contrarrestados. (Aragan, 2015), hace un señalamiento interesante con relación a los negocios, ya que al no tener una buena publicidad y al no informarse acerca de las nuevas alternativas publicitarias, presentan desventajas comerciales en el sector urbano, convirtiéndose en un obstáculo para que los dueños de los negocios no puedan emprender. Según (Vega, et al, 2017) existe un grave problema de mortandad dentro de las pequeñas y medianas empresas (Pymes), dando pie a que se plantee el hecho de utilizar medios alternativos publicitarios como una herramienta de difusión comercial que les auxilie a incrementar sus ventas dentro de un periodo de tiempo determinado. (Uribe \&
Sabogal, 2020), defina a Instagram como la red social más importante para generar ventas en las pymes, debido a la amplia variedad de formatos que ofrece, publicación de fotos sencillas o en carrusel, historias, etc.

Según (Vega, et al, 2017) los usuarios expuestos se sintieron más cercanos a la marca, se sugiere apoyar la fidelización de estos y genera una publicidad de boca en boca.

\subsection{La importancia de los proveedores}

Un proveedor tiene una capacidad de producción para un período de tiempo determinado (Ruiz, et al. 2013). Para (Sandoval, 2018) la relación con proveedores ha sido ampliamente estudiada en el campo de la cadena de suministro en las últimas tres décadas. Según (Ríos, et al., 2015), se debe seleccionar de una base de proveedores competitiva es de alta importancia en la búsqueda de mejores resultados.

El desempeño del proveedor no sólo está determinado por su participación en programas de desarrollo sino también por su perfil de capacidades de absorción, de asociación y su nivel de compromiso con el cliente (Ríos, et al., 2015). El éxito de la organización depende de una muy buena selección de proveedores y es una tarea crítica conseguir los diferentes objetivos de la cadena de suministro (Blanco 2016). Seleccionar el mejor proveedor en un problema de decisión en presencia de múltiples atributos, el cual ha sido resuelto hasta el momento de forma empírica y teniendo en cuenta la experiencia de los especialistas encargados de la actividad (González \& Garza, 2003).

Para (Vílchez, 2003) destaca que los costos no son el único aspecto en que los proveedores influyen de manera determinante en los resultados de las empresas.

\section{MÉTODO}

La presente investigación hace uso del método inductivo, atendiendo de lo particular a lo general, se observan y comparan distintos fenómenos que ayudan a llegar a una respuesta concreta. Además, implica una investigación explicativo-descriptiva, teniendo en cuenta una serie de datos recabados. Posteriormente se organizan e interpretan para arrojar un 
conjunto de resultados. De igual forma se hace uso del método cuantitativo, por lo que fue necesaria la realización de una encuesta a 122 dueñas y dueños de pequeños negocios de distintos giros en la ciudad de San Pedro de las Colonias y ejidos a sus alrededores, esto con relación a las problemáticas actuales a las que se podrían estar enfrentando. La encuesta contiene 14 ítems que presentan de manera clara dichas problemáticas, a las que los dueños de los negocios dieron respuesta. Cada uno de estos ítems podrían ser respondidos con una de las siguientes cuatro respuestas: "totalmente de acuerdo", "de acuerdo", "en desacuerdo" y "totalmente en desacuerdo".

Con apoyo del Alfa de Cronbach se determinó que el instrumento era confiable.

$$
\left.\alpha=\frac{K}{K-1}\left[1-\frac{\sum S_{i}{ }^{2}}{S_{T}{ }^{2}}\right]\right]
$$

La siguiente tabla muestra el concentrado de respuestas para validar estadísticamente el instrumento.

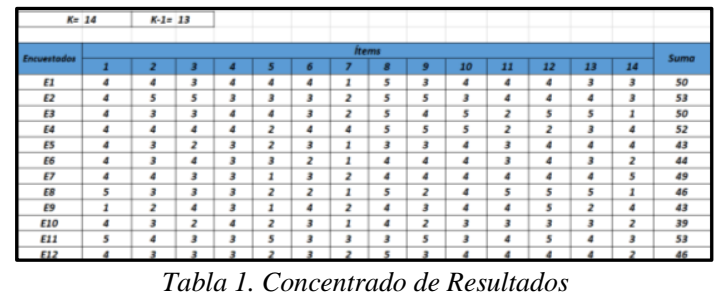

Se utilizó el Alfa de Cronbach, dando como resultado 0.86

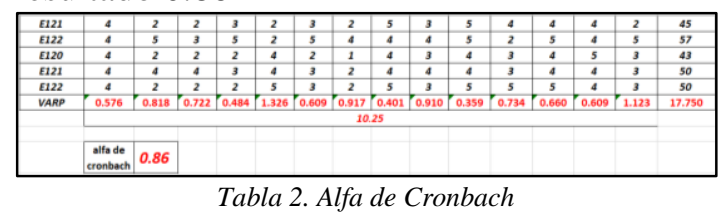

La Tabla 3 muestra el uso del coeficiente de correlación, permitiendo verificar la relación entra cada ítem.

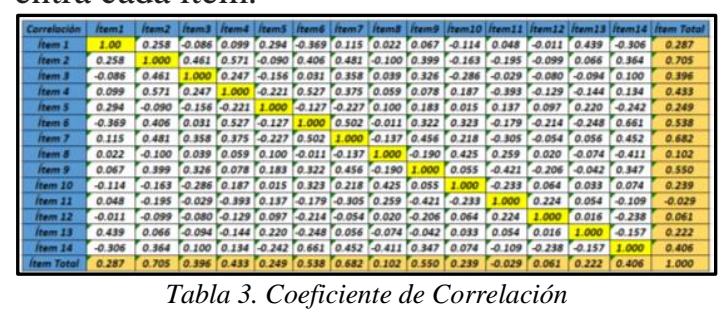

\section{RESULTADOS}

Debido a la pandemia, la mayoría de las encuestas fueron realizadas en línea, mediante un link que se proporcionó por redes sociales directamente a los propietarios de los negocios, sin embargo, algunas tuvieron que ser realizadas físicamente. A continuación, se presentan los resultados obtenidos en cada ítem.

La Fig. 1.1, 1.2 y 1.3 presentan todos los distintos giros de las 122 empresas que fueron consultadas, como se observa, hay una concentración alta de negocios de venta de comida, siendo 38 de los encuestados; le siguen 15 misceláneas, 9 papelerías, 7 negocios de venta de ropa, 5 panaderías, 4 carnicerías, entre otros como tortillerías, gimnasios, depósitos, fruterías, etc.

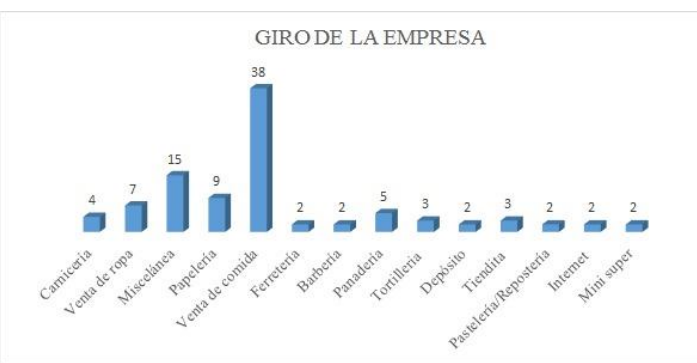

Figura 1.1. (Elaboración propia). Giro de la empresa.

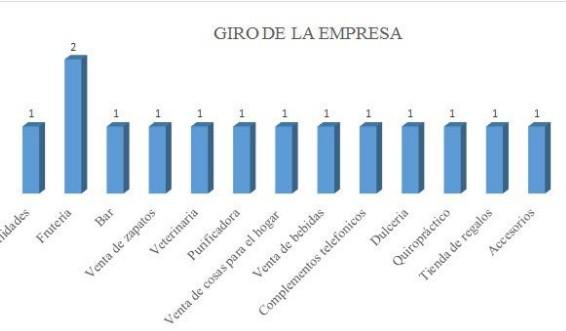

Figura 1.2. (Elaboración propia). Giro de la empresa.

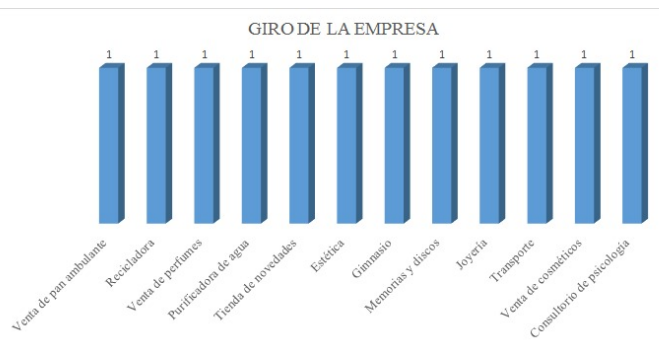

Figura 1.3. (Elaboración propia). Giro de la empresa.

La Fig. 2 plantea la falta de recursos económicos como un problema al que se enfrentan las pequeñas empresas. El 39.3\% respondió "totalmente de acuerdo", el $38.5 \%$ "de acuerdo", el 18\% "en desacuerdo" y el 
$4.2 \%$ "totalmente en desacuerdo". Efectivamente, el $77.8 \%$ de los negocios presentan problemas por la falta de recursos económicos en sus negocios.

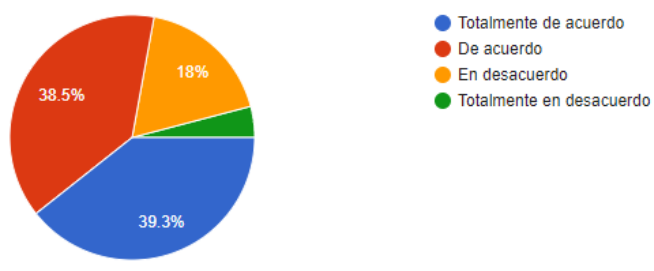

Figura 2. (Elaboración propia). Recursos económicos.

La Fig. 3 hace referencia a la publicidad utilizada en los pequeños negocios y sobre si es suficiente para realmente ayudar a sus ingresos. El 45.9\% respondió "de acuerdo", el $35.2 \%$ "totalmente de acuerdo", el 13.1 "en desacuerdo" y el 5.8\% "totalmente en desacuerdo". El $81.1 \%$ de los encuestados concuerdan en que la publicidad que realizan es suficiente.

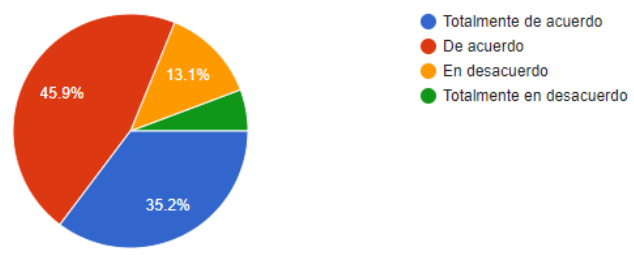

Figura 3. (Elaboración propia). Publicidad.

La Fig. 4 analiza si la ubicación en la que se encuentran estos negocios influye de alguna manera en sus ventas. El 46.7\% respondió "en desacuerdo", el $29.5 \%$ "totalmente en desacuerdo", el 14.8\% "de acuerdo" y el 9\% "totalmente de acuerdo". En este caso la ubicación no es una problemática para el $79.2 \%$ de los encuestados.

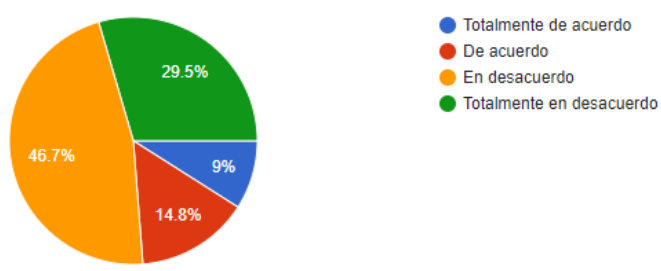

Figura 4. (Elaboración propia). Ubicación.

La Fig. 5 explica si la competencia con negocios cercanos influye de manera negativa en las pequeñas empresas. El 37.2\% respondió "en desacuerdo", el 24\% "de acuerdo", el $19.8 \%$ "totalmente en desacuerdo" y el $19 \%$ "totalmente de acuerdo". Se puede observar que la diferencia no es muy significativa, el $57 \%$ no creen que otros negocios los afecten, sin embargo, el $43 \%$ sí consideran que los afectan. Una persona se abstuvo de responder.

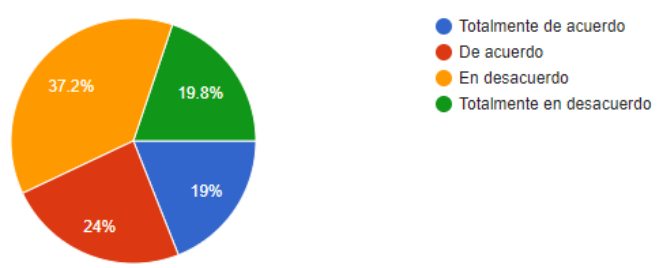

Figura 5. (Elaboración propia). Competencia.

La Fig. 6 infiere en si las ventas bajas son un factor para que los negocios no obtengan tantos ingresos. El $45.9 \%$ respondió "de acuerdo", el 38.5\% "totalmente de acuerdo", el $8.2 \%$ "en desacuerdo" y el 7.4\% "totalmente en desacuerdo". Es claro que el $88 \%$ considera que las ventas bajas repercuten sus ingresos.

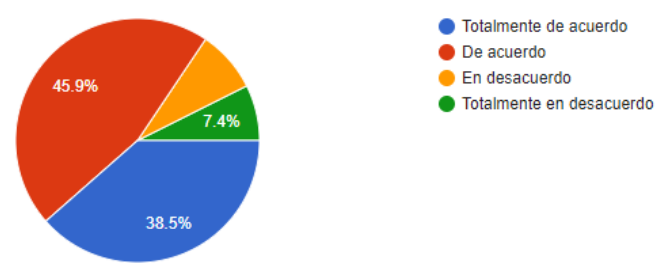

Figura 6. (Elaboración propia). Ventas bajas.

La Fig. 7 indica los resultados relacionados con el local en el que encuentran los negocios, y si estos son suficientes para la cantidad de personas que recibe cada uno. El $41 \%$ respondió "de acuerdo", el $34.4 \%$ respondió "totalmente de acuerdo", el $21.3 \%$ "es desacuerdo" y el 3.3\% "totalmente en desacuerdo". De acuerdo a esto es claro que, para el $75.4 \%$ de los dueños, su local no es inconveniente, por el contrario, es adecuado para la cantidad de clientes que reciben. 


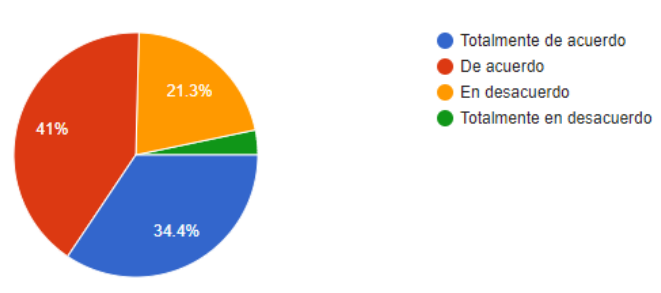

Figura 7. (Elaboración propia). Espacio del local.

La Fig. 8 muestra si la situación económica de la ciudad de San Pedro es un impedimento para el crecimiento de los negocios. El 46.7\% respondió "totalmente de acuerdo", el 32\% "de acuerdo", el 18.9\% "en desacuerdo" y el 2.4\% "totalmente en desacuerdo". El 78.7\% opina que su economía se ve afectada directamente por la de la ciudad.

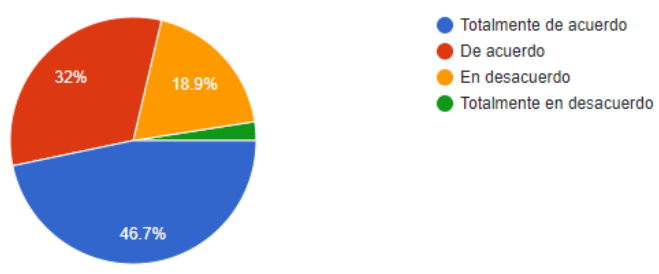

Figura 8. (Elaboración propia). Economía de la ciudad.

La Fig. 9 expresa si los permisos requeridos que otorga el municipio representan dificultades para los encuestados. El 30.3\% respondió "de acuerdo", el 27\% "en desacuerdo", el 22.1\% "totalmente de acuerdo y el " $20.5 \%$ "totalmente en desacuerdo". De nuevo no es mucha la diferencia, aunque el $52.4 \%$ considera que estos permisos sí son un inconveniente.

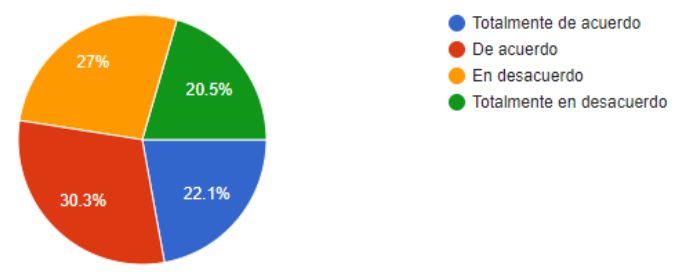

Figura 9. (Elaboración propia). Permisos del municipio.

La Fig. 10 plantea si la falta de personal es un problema para los comerciantes. El $38.5 \%$ respondió "en desacuerdo", el 28.7\% "de acuerdo", el 17.2\% "totalmente de acuerdo" y el 15.6\% "totalmente en desacuerdo". Por muy poca diferencia, el $54.1 \%$ de las personas consideran que la falta de personal no les afecta significativamente.
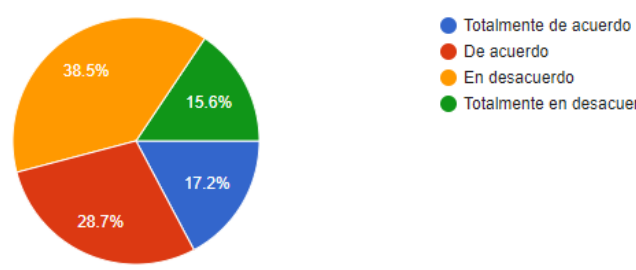

Figura 10. (Elaboración propia). Falta de personal.

La Fig. 11 considera el poco conocimiento sobre el cómo planear o manejar un negocio por parte de los dueños de los mismos y si esto ha sido un factor determinante para cumplir con sus objetivos. El 40\% respondió "en desacuerdo", el 32.5\% “de acuerdo", el 15.8\% "totalmente en desacuerdo" y el $11.7 \%$ "totalmente de acuerdo". El 55.8\% creen que tienen un buen manejo de su negocio. Dos personas no respondieron este ítem.
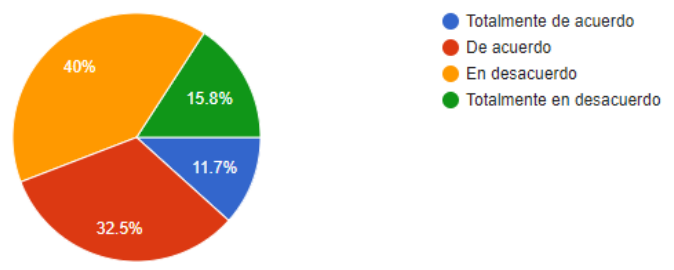

Figura 11. (Elaboración propia). Falta de planeación.

La Fig. 12 analiza si la falta de un local externo representa un inconveniente al querer expandir el negocio. El $42.6 \%$ respondió "en desacuerdo", el 21.3\% "totalmente de acuerdo", el 19.7\% "totalmente en desacuerdo" y el 16.4\% "de acuerdo". El $62.3 \%$ concuerdan en que no les preocupa rentar algún otro local.
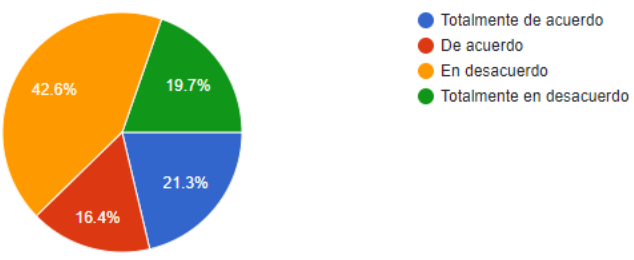

Figura 12. (Elaboración propia). Rentar un local.

La Fig. 13 presenta como problemática la falta de mobiliario para atender a los clientes. El $37.8 \%$ respondió "totalmente de acuerdo", el $35.3 \%$ "de acuerdo", el $21.8 \%$ "en 
desacuerdo" y el 5.1\% "totalmente en desacuerdo". El $73.1 \%$ de los encuestados opinan que cuentan con el mobiliario suficiente para atender su demanda. 3 personas no contestaron este ítem.

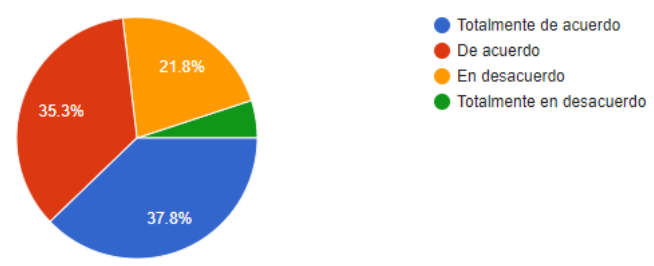

Figura 13. (Elaboración propia). Mobiliario.

La Fig. 14 plantea si la falta de producto por proveedores es un inconveniente al momento de abastecer o suministrar los negocios. El $36.9 \%$ respondió "en desacuerdo", el 36.1 "de acuerdo", el 17.2\% "totalmente de acuerdo" y el 9.8\% "totalmente en desacuerdo". El 53.3\% de las personas que contestaron afirman que tienen problemas con proveedores.
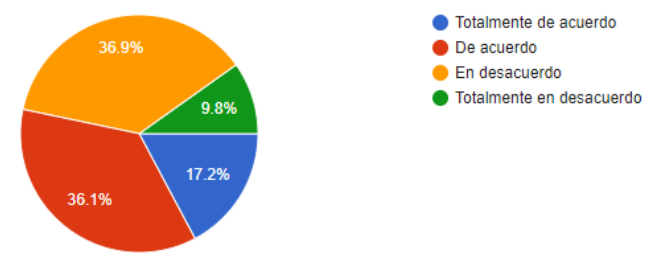

Figura 14. (Elaboración propia). Proveedores.

La Fig. 15 expone sin duda alguna la problemática más seria actualmente la pandemia por el COVID-19. El $63.1 \%$ respondió "totalmente de acuerdo", el 26.2\% "de acuerdo", aproximadamente el 8\% y $2 \%$ respondieron "en desacuerdo y "totalmente en desacuerdo" respectivamente. Claramente el 89.3\% de los dueños de estos pequeños negocios coinciden en que es un problema que afecta no solo a los comerciantes, sino a todos en general.
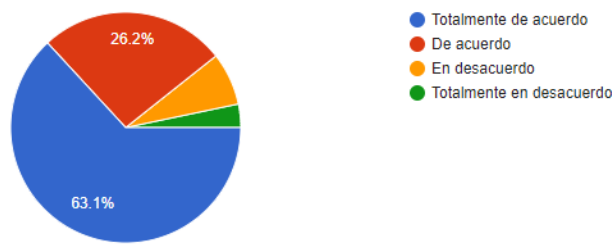

Figura 15. (Elaboración propia). Pandemia.

\section{CONCLUSIONES}

Después de analizar los resultados se puede concluir que a pesar de que los dueños de los pequeños negocios no tienen estudios o conocimientos académicos sobre cómo administrar una empresa, el 55.8\% de 122 negocios logran que estos se sostengan con el paso del tiempo, pues las problemáticas que más los afectan son aspectos que no están a su alcance, o que no dependen del negocio en sí para solucionarse, como lo serían: los recursos económicos, la economía de la ciudad, los permisos con el municipio y la más notoria en la actualidad, la pandemia; algunos de los problemas que sí podrían solucionar por su cuenta son las ventas bajas y la relación con los proveedores.

Los dueños afirman tener una planeación adecuada para satisfacer los objetivos de sus respectivos negocios; debido a que no presentan inconvenientes en el resto de las problemáticas, por ejemplo: la publicidad. La mayoría de los negocios opinan que la publicidad que realizan es la suficiente para darse a conocer, en este caso realmente no existe evidencia de dicha publicidad, tales como carteles, anuncios en el periódico o por redes sociales; se teoriza que la manera en que se introducen al mercado es por medio de los clientes, con el servicio que brindan y las relaciones que crean con estos, así ellos recomiendan el negocio a sus conocidos, lo cual indica que la publicidad que ayuda los dueños de los establecimientos es a través del método de boca en boca.

Incluso teniendo una buena planeación y contar con la publicidad suficiente no se le da tanta prioridad a otros aspectos, como lo es con los proveedores, esto podría depender de distintos aspectos, los proveedores no cuentan con la mercancía necesaria, en la relación calidad-precio de sus productos, el tiempo en el que lo solicitan, etc. Entonces, se concluye que la hipótesis se cumple parcialmente, ya que a la relación de la planeación con la publicidad se le da más relevancia que a la relación con los proveedores. Sin embargo, depende del criterio de cada dueño la prioridad que se le otorga a cada problema. 


\section{REFERENCIAS}

E. Reyes. (2019). "Nivel de competencia de planeación y gestión que tienen los pequeños negocios dedicados a la comercialización en el Estado de Nayarit". Revista Edúcate con ciencia. Volumen 24. No. 25. ISSN: 2007-6347. Tepic, Nayarit. México. Pp. $72-89$. http://tecnocientifica.com.mx/educateconciencia/index.php/revistaeducate/article/download/4 $3 / 24$

M. Augusta-Moreta. (2018). "Planeación estratégica en PYMES: limitaciones, objetivos y estrategias". UTCiencia "Ciencia y Tecnología al servicio del pueblo". http://investigacion.utc.edu.ec/revistasutc/index.php/utciencia/article/view/83/79

J. A. Correa, L. J. Ramírez, C. E. Castaño. (2010). "La importancia de la planeación financiera en la elaboración de los planes de negocio y su impacto en el desarrollo empresarial". Revista Facultad de Ciencias Económicas: Investigación y Reflexión, vol. XVIII, núm. 1 pág. 179-194 Universidad Militar Nueva Granada Bogotá, Colombia. https://www.redalyc.org/pdf/909/90920479010.pdf

F. Madrigal, S. Madrigal, C. Guerrero. (2015). "Planeación Estratégica y gestión del conocimiento en las pequeñas y medianas empresas, (Pymes), herramientas básicas para su permanencia y consolidación". https://core.ac.uk/download/pdf/236412838.pdf

G. Velasco. (2020). "Competitividad empresarial. Administración de Pequeños Negocios y Emprendimiento". http://cathi.uacj.mx/handle/20.500.11961/17946;jsessionid=20418C77317CA3BB7D2F4A5E 01734212

M. Augusta-Moreta. (2018). "Planeación estratégica en PYMES: limitaciones, objetivos y estrategias". UTCiencia "Ciencia y Tecnología al servicio del pueblo". http://investigacion.utc.edu.ec/revistasutc/index.php/utciencia/article/view/83/79

J. A. Correa, L. J. Ramírez, C. E. Castaño. (2010). "La importancia de la planeación financiera en la elaboración de los planes de negocio y su impacto en el desarrollo empresarial". Revista Facultad de Ciencias Económicas: Investigación y Reflexión, vol. XVIII, núm. 1 pág. 179-194 Universidad Militar Nueva Granada Bogotá, Colombia. https://www.redalyc.org/pdf/909/90920479010.pdf

F. Madrigal, S. Madrigal, C. Guerrero. (2015). "Planeación Estratégica y gestión del conocimiento en las pequeñas y medianas empresas, (Pymes), herramientas básicas para su permanencia y consolidación". https://core.ac.uk/download/pdf/236412838.pdf

G. Velasco. (2020). "Competitividad empresarial. Administración de Pequeños Negocios y Emprendimiento".

http://cathi.uacj.mx/handle/20.500.11961/17946;jsessionid=20418C77317CA3BB7D2F4A5E 01734212

A. Araya. (2017). "Modelos de planeación estratégica en las empresas familiares". Tec Empre. [en línea]., vol.11, n.1, pp.23-34. https://www.scielo.sa.cr/scielo.php?pid=S1659-33592017000100023\&script=sci_arttext

M. Larrañaga, M. T. Ortega. (2018). "La planeación estratégica de las micro y pequeñas empresas de alojamiento temporal en Cuernavaca, Morelos, México”. Universidad Politécnica del Estado de

Morelos. http://www.web.facpya.uanl.mx/Vinculategica/vinculat\%C3\%A9gica_2/42\%20LARRA+\%C 3\%A6AGA_ORTEGA.pdf

A. Terrones-Cordero. (2013). "Planeación participativa para elaborar un plan de desarrollo municipal: el caso de Acaxochitlán, Hidalgo”. Economía, sociedad y territorio. XIII (42.521-559.). ISSN: $1405-8421$.

J. A. Valdivia. (2015). "Comercialización de productos y servicios en pequeños negocios o microempresas". $\quad$ IC $\quad$ Editorial. $\quad$ ISBN: 978-84-16351-60-2. 
https://books.google.es/books?hl=es\&lr=\&id=y8LIBgAAQBAJ\&oi=fnd\&pg=PT4\&dq=publi cidad+en+los+peque\%C3\%B1os+negocios\&ots=kpx7QRxnqv\&sig=laKMbhKSMwtFXqzJ XU8fkr81Jes

J. D. Fernández. (2005). “Algunas reflexiones en torno al problema de la creación de necesidades en publicidad y marketing”. Comunicación: revista internacional de comunicación audiovisual, publicidad y estudios culturales, 1 (3), 101-124. https://idus.us.es/handle/11441/12980

F. Cabezuelo. (2014). "Una propuesta científica para el estudio de la eficacia publicitaria. Fundamentos de la eficacia publicitaria y retorno de la inversión". Guardia Delta Publicaciones / Empresa, Madrid 2012. ICONO 14, Revista de comunicación y tecnologías emergentes, 12(1) ,497-498.

E. J. Barillas. (2010). "Estrategias alternativas de publicidad para los negocios de antigua Guatemala". Facultad de Ciencias Económicas y Empresariales de la Universidad del Istmo. Guatemala. [En línea]. http://glifos.unis.edu.gt/digital/tesis/2010/25916.pdf

G. J. Tellis., I. Redondo. (2002). "Estrategias de publicidad y promoción”. Pearson educación, Capitulo 5-7. S.A. Madrid.

F. M. Aragón. (2016). "Análisis de la incidencia de la publicidad multimedia en los locales comerciales y negocios de la zona urbano - céntrica del cantón Antonio ante". Trabajo de Grado, previo a la obtención del título de Licenciado en la especialidad de Diseño Gráfico. http://repositorio.utn.edu.ec/bitstream/123456789/5130/1/05\%20FECYT\%202696\%20TRAB AJO\%20GRADO.pdf

A. Vega, et al. (2018). "La publicidad BTL como estrategia para fomentar la consolidación de las Pymes".

$36-52$.

https://www.cadernomarketingunimep.com.br/ojs/index.php/cadprofmkt/article/view/88/100

R. Flores, I. Flores, M. Vázquez. (2015). "La publicidad, una estrategia de éxito para las micro, pequeñas y medianas empresas de la región centro y suroeste del Estado de Hidalgo". $\begin{array}{lll}\text { INQUIETUD } & \text { EMPRESARIAL, } & 133-152 .\end{array}$ https://revistas.uptc.edu.co/index.php/inquietud_empresarial/article/view/3344

C. I. Uribe, D. F. Sabogal. (2021). "Marketing digital en micro y pequeñas empresas de publicidad de Bogotá. Revista Universidad Y Empresa”. 23(40). https://doi.org/10.12804/revistas.urosario.edu.co/empresa/a.8730

A. J. Ruiz, A. Mendoza, J. H. Ablanedo. (2013). "Modelo para la planificación en la cadena de suministro: Selección y asignación a proveedores en el caso de lotes fijos". Ingeniería y Desarrollo, 31(1),1-21. ISSN: 0122-3461.

M. Sandoval, A. Gianmarco. (2018). "Propuesta de indicador para la evaluación de la relación con proveedores en mypes en Piura". [En línea] Universidad de Piura. https://pirhua.udep.edu.pe/handle/11042/3461

A. Vílchez. (2003). "Gestión de inventario. Relación con los proveedores en franquicias de comida rápida”. Revista Venezolana de Gerencia. Vol. 8, núm. 23. Pp. 510-525. ISSN: 1315-9984

N. J. Ríos, A. Arellano, E. Coronado. (2015). "Evaluación del desempeño de proveedores en pequeñas empresas del sector servicios”. Pág. 1-22. Universidad Juárez del Estado de Durango, Durango, Durango,

México. http://acacia.org.mx/busqueda/pdf/EVALUACION_DEL_DESEMPENO_DE_PROVEEDO RES_EN_PEQUENAS_EMPRESAS_DEL_SECTOR_SERVICIOS.pdf

J. D. Blanco. (2016). "Diseño del sistema de gestión de evaluación de proveedores de la industria petrolera en la empresa petrotiger ltad, basado en la aplicación de la norma iso 9001: 2015 y ntc ohsas 18001: 2007 (tesis de pregrado)". http://repository.ucc.edu.co/handle/ucc/8067

A. González, R. Garza. (2003). "Aplicación de las técnicas multicriteriales en la evaluación y selección de proveedores”. Ingeniería Industrial, ISSN-e 1815-5936, Vol. 24, № 2. https://dialnet.unirioja.es/servlet/articulo?codigo $=4786742$

W. A. Sarache, C. Hoyos, J. C. Burbano J. (2004). "Procedimiento para la evaluación de proveedores mediante técnicas multicriterio". Scientia Et Technica, vol. X, núm. 24, pp. 219-224. 
Universidad Tecnológica de Pereira. Pereira, Colombia. https://www.redalyc.org/pdf/849/84912053040.pdf 